This is a preprint version of the following paper:

A. Zameshin, I.A. Makhotkin, S.N. Yakunin, R.W.E. van de Kruijs, A.E. Yakshin, F. Bijkerk, Reconstruction of interfaces of periodic multilayers from X-ray reflectivity using a free-form approach, J. Appl. Crystallogr. 49 (2016) 1300-1307. doi:10.1107/S160057671601044X.

\title{
Reconstruction of interfaces of periodic multilayers from X-ray reflectivity using free-form approach
}

Authors

Andrey Zameshin ${ }^{\text {a* }}$, Igor A. Makhotkin ${ }^{a}$, Sergey N. Yakunin ${ }^{\mathrm{b}}$, Robbert W. E. van de Kruijs ${ }^{\mathrm{a}}$, Andrey E. Yakshin ${ }^{a}$ and Fred Bijkerk ${ }^{a}$

andustrial Focus Group XUV Optics, MESA+ Institute for Nanotechnology, University of Twente, Drienerlolaan 5, Enschede, 7522 NB, The Netherlands

${ }^{\mathrm{b}}$ NRC Kurchatov Institute, Moscow, Russian Federation

Correspondence email: a.zameshin@utwente.nl

Synopsis A custom free-form approach is used for reconstruction of GIXRR from periodic multilayers, simulated and experimental data fits are presented.

Abstract Grazing Incidence X-ray Reflectivity (GIXRR) is a widely used analysis method for thin films and multilayer structures. However, conventional so-called model-based approaches of structural reconstruction from GIXRR data are lacking analytical power when dealing with very thin structures (down to the nm scale), because a priori assumptions have to be made about the interface composition and structure. This makes it very difficult to reliably analyse such structures. In this work custom freeform approach is presented which solves this task without the need for a priori assumptions on layer or interface parameters. As a proof of principle, a perfect optical constant profile reconstruction and GIXRR curve matching for the simulated data are demonstrated. The developed approach is used to analyse structures of multilayer LaN/B Bragg reflectors designed for the Extreme UV range. Performed analysis allowed to reveal the difference in optical constant profiles of these structures produced with different processes. The uncertainties of structural reconstruction are also discussed.

Keywords: X-Ray reflectivity; free-form; model-independent; multilayer; EUV lithography 


\section{Introduction}

Grazing incidence X-ray reflectivity (GIXRR) is a widespread technique to investigate thin film and layered structures (Tolan, 1999)(Daillant \& Gibaud, 2009). The main method of the analysis of GIXRR data is based on creating a model of a structure and then fitting calculated reflectivity curves to the measured data by varying model parameters, e.g. layer thicknesses, layer densities, but also the size of interfacial regions and roughness (Windt, 1998). This analysis method will be referred further as model-based technique. Interface roughness/diffusion can be presented by either Debye-Waller and Nevot-Croce models (Daillant \& Gibaud, 2009), or by fixed shape gradual transition sublayers (graded interfaces) (Windt, 1998). This means that prior to the fitting one has to assume a certain model of the structure, including the sequence of the layers, and the presence and description of interfaces.

Model-based techniques perform well for thicker layers, where the contribution of interfaces is relatively small compared to contribution of layers. An example of such periodic structures are $\mathrm{Mo} / \mathrm{Si}$ based multilayers with a period of about $7 \mathrm{~nm}$, which were extensively studied with various model approaches (Yakshin et al., 2000)(Jergel et al., 1998). An example of a contrary case are La/B-based multilayers with a period of about $3 \mathrm{~nm}$ where the interfaces were found to be comparable to the layer thicknesses (Makhotkin, 2013). For such multilayer structures selection of the shape of interfaces will determine the outcome of the reconstruction. At the same time reconstruction of $\mathrm{La} / \mathrm{B}-\mathrm{b}$ ased structures is needed in view of the potential application of these structures for the next generation lithography, namely at around 6-7 $\mathrm{nm}(6 . \mathrm{xm})$ : the wavelength range denoted as beyond extreme ultraviolet (BEUV) (Platonov et al., 2002; Tsarfati et al., 2009; Banine et al., 2010; Makhotkin et al., 2012; Kuznetsov et al., 2015).

There are alternative model-independent approaches, which do not require a model in the sense described above. This is a class of methods which does not use a representation of the structure as a combination of layers and fixed-shape interfaces (Pedersen, 1992; Sanyal et al., 1993; Zhou \& Chen, 1993; Zimmermann et al., 2000). An important advantage of the model-independent approaches is that in this procedure the profile of the entire structure can be reconstructed in one fitting process. At the same time the conventional model-based approach would require iterative trying of different interface profiles and starting conditions, expecting that the right interface type is included in the model. In fact for complex structures it becomes increasingly difficult to make a proper guess for the type of the interface profile, and this makes the model approach reconstruction a very time consuming task or not feasible at all.

Most commonly the model-independent approaches use kinematic theory. However, dealing with high-reflectance multilayers requires a dynamical theory (Daillant \& Gibaud, 2009) that allows correct analysis of strong multilayer diffraction peaks. Previous attempts to do so were quite 
complicated (Zimmermann et al., 2000), but the most suitable solution for our task is to make a modification of a free-form methods, which explicitly present structure as a set of sublayers (Zhou \& Chen, 1993)(Kozhevnikov, 2003). The calculation of GIXRR with such approaches can be easily done with the dynamical theory, and there are no obstacles to make them work with the periodic structures.

In this article we will present the first attempt of a free-form approach for the reconstruction of optical constant profile of the periodic multilayer structures based on rigorous description of reflectivity following the dynamic theory. With help of this approach we reconstructed the structures of two LaN/B multilayer mirrors. The LaN/B multilayer mirrors are one of the best reflectors for $6.7 \mathrm{~nm}$ wavelength and as was shown in (Makhotkin et al., 2012), their reflection strongly depends on the way the lanthanum nitride is fabricated. Recently Kuznetsov et all (Kuznetsov et al., 2015) have demonstrated that insertion of a thin La interlayer below LaN layer resulted in a record 64\% reflectance of a multilayer mirror at $6.7 \mathrm{~nm}$ wavelength and an incident angle of $1.5^{\circ}$ off-normal. Using free-form approach we analysed the structure of this multilayer mirror and discuss the influence of deposition process on the structure of the interfaces.

\section{Free-form approach}

The main goal of GIXRR analysis of periodic multilayer films is to reconstruct the structure of its periodic part, because it determines the functional properties of the coating, e.g. optical or magnetic properties. The conventional model approaches present multilayer period as a set of layers, parametrized by individual layer thickness, layer material parameters, and thickness of interfaces, while the shape of interfaces is fixed. In the free-form analysis we divide the optical constant profile of multilayer structure into thin independent sublayers with the same fixed thickness. The optical constant of each individual sublayer is varied during the fitting of GIXRR data.

The thicknesses of the sublayers are determined by the size of the minimal resolvable feature $d_{\min }$ in a given GIXRR measurement. $d_{\min }$ is defined by the maximal scattering vector $q_{z(\max )}$, corresponding to the last observed Bragg peak:

$d_{\min }=\frac{1}{2} \frac{2 \pi}{q_{z(\max )}}$.

Similar estimate was obtained earlier in (Chou et al., 1997). In our work $d_{\min }$ defines the optimal number of sublayers $N_{\Lambda}=\Lambda / d_{\min }$ in a structure with a period thickness $\Lambda$.

For a multilayer structure, containing two alternating materials $A$ and $B$ with refractive indexes $n_{A}$ and $n_{B}$ the optical constant profile of its period can be described as $n_{i}=n_{A}+x_{i}\left(n_{B}-n_{A}\right)$, where $x_{i}$ is the concentration of material B in material A in the $i$-th sublayer. Therefore the optical constant 
profile of the multilayer can be determined by vector $x$ of $x_{i}$ values, while $n_{A}$ and $n_{B}$ are fixed, so the space of solutions becomes defined by their values. The refractive index $n$ is a complex value and is in the $\mathrm{X}$-ray region usually shown as $n=1-\delta+i \beta$. In our calculations we take into account not only the real part of the optical constant profile, but also the imaginary part, automatically satisfying the Kramers-Kronig relation between the real and imaginary parts. Therefore, each sublayer represents a mixture of materials $A$ and $B$. The multilayer period now can be parameterized with $\boldsymbol{P}=$ $(\boldsymbol{x}, \Lambda)$ where $\Lambda$ is the bilayer thickness. Although all periods in the periodic multilayer are considered identical, the top and bottom bilayers can be different because of interaction with environment and substrate. The full space of fitting parameters is $\boldsymbol{P}=\left(\boldsymbol{x}_{\text {top }}, L_{t o p}, \boldsymbol{x}_{\text {per }}, \Lambda, \boldsymbol{x}_{\text {bot }}, L_{b o t}\right)$, where $\boldsymbol{x}_{\text {top }}$, $\boldsymbol{x}_{\text {per }}, \boldsymbol{x}_{\text {bot }}$ and $L_{\text {top }}, \Lambda, L_{\text {bot }}$ are defined and described above for the top, periodic, and bottom part correspondingly.

The optimization algorithm finds the final optical constant profile $\widetilde{\boldsymbol{P}}$ which satisfies the condition $\chi^{2}(\widetilde{\boldsymbol{P}})=\min \chi^{2}(\boldsymbol{P})$.

Here $\chi^{2}$ is the goodness of the fit value presented as a sum of squares of residuals of the measured and simulated GIXRR $u(\theta, \boldsymbol{P})$ as described it (Yakunin et al., 2014) and is defined as

$\chi^{2}=\frac{1}{N-l}\left(\sum_{\theta} u^{2}(\theta, P)\right)$

$u(\theta, \boldsymbol{P})=\frac{I_{\text {calc }}(\theta, \boldsymbol{P})-I_{\text {exp }}(\theta)}{\sigma(\theta)}$,

where $N$ is a number of measured data points, $\theta$ is angle of incidence in GIXR measurements and $I_{\text {calc }}(\theta, \boldsymbol{P})$ - calculated intensity of GIXRR, $I_{\text {exp }}(\theta, \boldsymbol{P})$ - measured intensity of GIXRR, $\sigma(\theta)$ uncertainty of GIXRR measurement, $l$ - number of the fit parameters.

In a realistic multilayer structure, the optical constant profile is a smooth function and for this reason no large jumps in $n_{i}$ should be allowed from one sublayer to the next one. To incorporate this smoothness in the optimization procedure, a regularization coefficient $R_{\text {reg }}$ is added to the $\chi^{2}$ merit function $\tilde{\chi}^{2}=\chi^{2}+R_{\text {reg }}$.

$R_{r e g}=r \sum_{i=2}^{N_{\Lambda}-1}\left(2 x_{i}-x_{i-1}-x_{i+1}\right)^{2}+r\left(2 x_{1}-x_{2}-x_{N_{\Lambda}}\right)^{2}+r\left(2 x_{N_{\Lambda}}-x_{N_{\Lambda}-1}-x_{1}\right)^{2}$,

here $N_{\Lambda}$ is the length of the vector $\boldsymbol{x}, r$ is a small number - the regularization parameter that specifies the degree of profile smoothness. The first term governs the continuity within the period, and the second and third set continuity on its borders. The same smoothening was applied for the top and bottom part of the structure.

For the X-ray reflectivity simulation we used the Abeles matrix approach optimized for modelling of the periodic multilayer structures. In order to solve the optimization problem of Eq. (2) a Levenberg- 
Marquardt algorithm was used. Standard deviations of reconstructed parameters were calculated by the least squares method as in (Yakunin et al., 2014).

In example of $\mathrm{La} / \mathrm{B}$-based multilayers long GIXRR measurement in $\mathrm{CuK} \alpha 1$ spectrum has Bragg peaks appearing until $\theta=7^{\circ}$, so the value of $d_{\min }$ is approximately $0.3 \mathrm{~nm}$, based on Eq. (1). To describe such multilayer with a period thickness $\Lambda=3.5 \mathrm{~nm}$ one should use around 11-12 sublayers. The amount of iterations needed for finding an optimal solution is between 100-200.

The fitting procedure starts with all of the sub-layers having the same optical constants defined as $x=$ 0.5. Due to the presence of sharp Bragg peaks the algorithm converges early on the oscillatory structural profiles, and later only refines these profiles. An example of the typical evolution of the $\delta$ profile during the fit is shown in Fig. 1. In this figure, as in any following figures in this paper, only the decrement of the real part of the refractive index is shown as a $\delta$-profile, the imaginary part is omitted. The algorithm was run on a conventional Core i7-3520M CPU on a work station laptop, with two of its $2.9 \mathrm{GHz}$ cores being used. The fitting procedure shown was performed in 153 iterations and took 10 minutes in total. At iteration 35 the basic oscillating shape of the profile was already obtained, with a refinement being done within the remaining iterations. This procedure was sufficient to result in the high quality fit as the ones shown in the "Results and discussion" section.
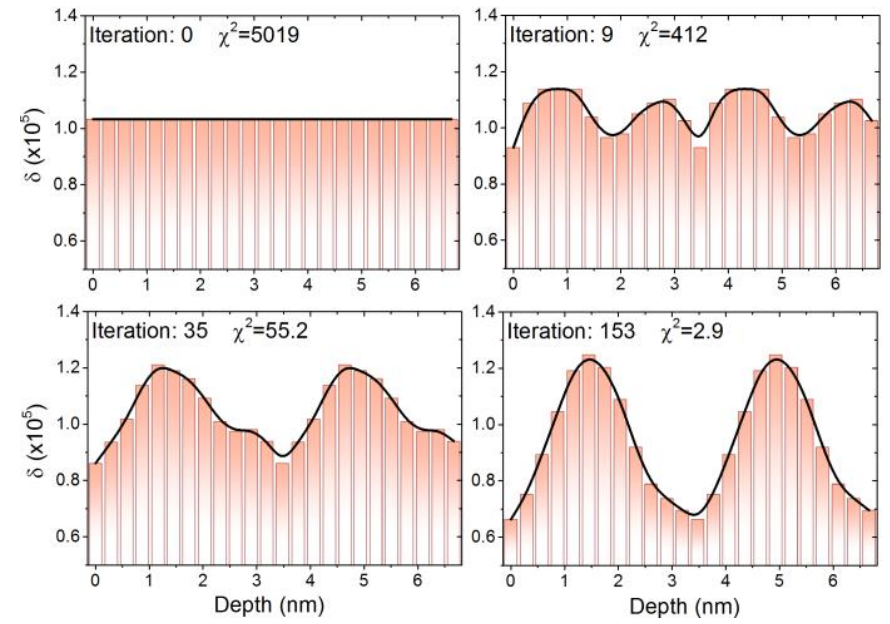

Figure 1 Evolution of $\delta$-profile during fit. Shown on each subplot are two consecutive periodic parts of the multilayer. Goodness of fit value $\left(\chi^{2}\right)$ by eq. (3) for each step is shown.

\section{Results and discussion}

\subsection{Fit of artificially generated data from a model system}

It is well known that the process of solving an inverse problem of GIXRR can result in more than one solution (Pershan, 1994)(Daillant \& Gibaud, 2009). It can be proven (Kozhevnikov, 2003) that even for a single layer film with sharp interfaces, four distinct electron density profiles can be found. For a 
periodic multilayer, different solutions are also possible (Zimmermann et al., 2000). To test the ambiguity of the reconstruction of GIXRR data with free form analysis, we have performed a numerical experiment on a simulated set of data.

We composed a model system corresponding to our best knowledge with $\mathrm{La} / \mathrm{B}$ multilayers, obtained by a model-based algorithm with interfaces modelled with transition layers taken from (Makhotkin, 2013). The structure consisted of 50 identical periods of alternating La and B layers with a non-ideal contrast between them, i.e. smaller contrast then between bulk materials. The optical properties of layers were such that La would allow a certain amount of B mixed in it, and vice versa. Furthermore the layers had wide asymmetrical interfaces. The structure was capped by a layer of B, covered with low density boron oxide. The profile was made of 200 sublayers in total and is shown as green curve in Fig. 2A. From this structure we calculated $\mathrm{Cu}-\mathrm{K} \alpha \mathrm{X}$-ray reflectivity by means of Abeles matrix formalism in angular span of $2 \theta$ from 0 till 7 degrees. An instrumental function was applied to the curve, which included a constant background of 0.05 counts/s; random stochastic noise with square root dependence on calculated intensity and realistic measurement time $\sqrt{I_{\text {calc }} / t}$; convolution with a Gaussian with a width of $0.01^{\circ}$ to account for broadening of the curve due to the limited instrumental resolution; and a modified total reflection region according to the real geometry of the measurement system. This made the simulated GIXRR look similar to experimental GIXRR in conditions described further in the section "Fit of experimental GIXRR curves".

In order to achieve different solutions of the same problem we varied different fitting parameters and starting conditions, namely regularization factor $r$, and starting $\boldsymbol{x}$ value as well as the number of sublayers in periodic part ( \pm 1 sublayer to optimum value). Each solution (or local minimum) was obtained with one run of the algorithm with chosen parameters and conditions. Then the original and reconstructed $\delta$-profiles were plotted together in Fig. 2A, and best GIXRR curves in Fig. 2B. The whole procedure results in two groups of $\delta$-profiles. Inside these groups deviations between individual $\delta$-profiles are very small and comparable to the minimum detectable feature size. Groups are marked as red and blue. There is no strict separation in fitting parameter space between these two groups. 

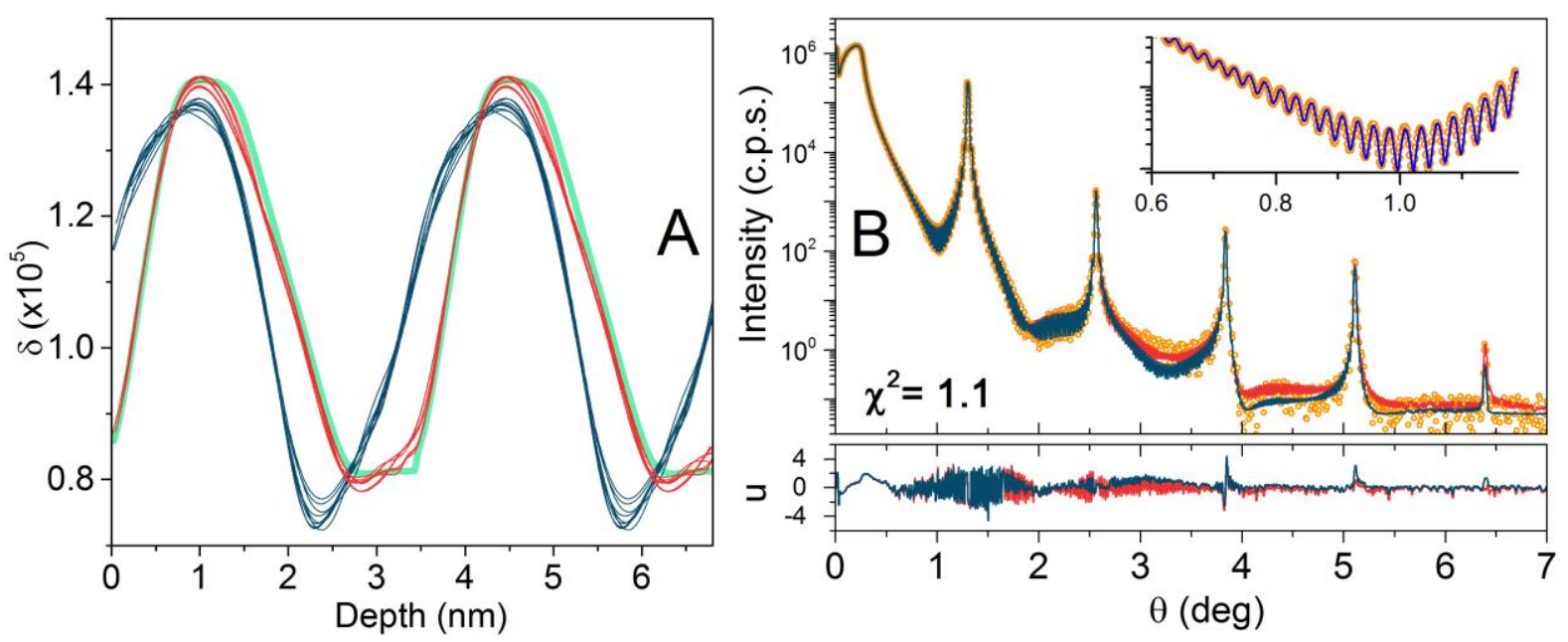

Figure 2 Fit of the GIXRR curve from artificially generated data with typical experimental limitations applied. A) Green $-\delta$-profile of the original model, red - the first group of solutions, blue - the second group of solutions. B) Top part: Yellow dots - original GIXRR curve from the generated data, red curve - best fit from the first group of solutions, blue - best fit from the second group of solutions. Bottom part: Red - residual between the original GIXRR and the best fit from the first group of solutions, blue - residual between original GIXRR and best fit from the second group of solutions.



Figure 3 Reproduction of $\delta$-profiles from Fig. 2A with the blue group of profiles inverted. Green curve $-\delta$-profile of the original model, red curves - the first group of solutions, blue - the second group of solutions with the inverted depth axis. After the inversion the interface slopes become almost the same for both groups of solutions.

The red group consists of solutions with the $\delta$-profiles being very close to the original profile. It has differences visible as distortions of the original profile, which are also within minimal detectable feature size. 
The blue group describes some of the features of the original model rather good, like general contrast and absolute ratio of the asymmetry of interfaces, but noticeably lowers the La and B regions, and inverts the slopes of interfaces. The inversion of slopes is demonstrated in Fig. 3, where inverted profiles from the blue group and original profiles from the red group are plotted. As seen they have almost perfect match of the interface slopes. This is in agreement with the known fact that reconstruction of GIXRR can be insensitive to the direction of interface asymmetry. Concerning the quality of the match between the calculated and original reflectivity curves, in the region around the second Bragg peak residuals of the red curve are objectively smaller than of the blue curve, because the latter solution has slightly shifted position of the Kiessig fringes. But still in general GIXRR curves seem very close to each other, therefore the separation between the two solutions cannot be reliably made if based only on GIXRR. This is a demonstration of uncertainly of GIXR technique that should be resolved by comparing the different solutions to existing preknowledge about the structure.

\subsection{Fit of experimental GIXRR curves}

Using the developed approach we have analysed two 50 period LaN/B multilayers with the period thickness of around $3.5 \mathrm{~nm}$, deposited by magnetron sputtering. The samples optimized for BEUV reflectance typically consist of 220 periods. However, the 50 period multilayers depend less on small period fluctuations from the deposition process and Kiessig fringes in their GIXRR are much easier to resolve, therefore they were used for the structural reconstruction.

The first sample was a conventional LaN/B multilayer, where $\mathrm{LaN}$ was obtained by reactive sputtering of La in $\mathrm{N}_{2}$ environment. X-Ray Photoelectron Spectroscopy of LaN/B multilayers (Kuznetsov et al., 2015) revealed the formation of BN compound during deposition of LaN on B. Since BN is optically unfavourable and has a negative influence on BEUV reflectance, a multilayer of $\mathrm{LaN} / \mathrm{La} / \mathrm{B}$ was made, with a $0.3 \mathrm{~nm}$ La interlayer deposited on top of B layer. This so called delayed nitridation approach resulted in a drastic improvement of the BEUV reflectivity of the full stack (220 period) multilayers, showing a reflectance increase from $58 \%$ to above $64 \%$ at $6.6 \mathrm{~nm}$ and an incident angle of $1.5^{\circ}$ off-normal. The same multilayer structure prepared with 50 periods was the second sample we studied in this paper and compared to the original LaN/B multilayer.

GIXRR data were measured using a PANalytical EMPYREAN X-ray diffractometer with monochromatized $C u K \alpha$ beam $(1.54056 \AA)$. The measurement time was 10 hours in the angular range of $\theta$ from 0 to $7^{\circ}$ with a step size of $0.002^{\circ}$ in the range of $0-1.5^{\circ}, 0.004^{\circ}$ in $1.5-2.7^{\circ}$ and $0.008^{\circ}$ in $2.7-$ $7^{\circ}$. This allowed to resolve Kiessig fringes at lower angles, while measuring longer at higher angles.

The measurements for both samples are shown in Fig. 4. There is a visible difference in the two curves, but it is mostly observed in the rate of the Bragg peak decay. It means that the structures are 
mostly similar, with only some small differences being present. To find them, a profile reconstruction was performed.

All instrumental parameters, namely angular and spectral resolution, direct beam intensity and the background were set accordingly to used experimental scheme of PANalytical Empyrean setup. Then the number of sublayers was varied in the range of \pm 1 sublayer and different starting conditions were applied to check the existence of possible different solutions. Indeed for both samples a manifold of solutions was obtained. Each solution was obtained in one run of the algorithm. All of them have a very good match with the experimental reflectivity curves with $\chi^{2}$ values around 3. Fig. 5 shows GIXRR curves for the solutions with 11 sublayers for each sample as an example.

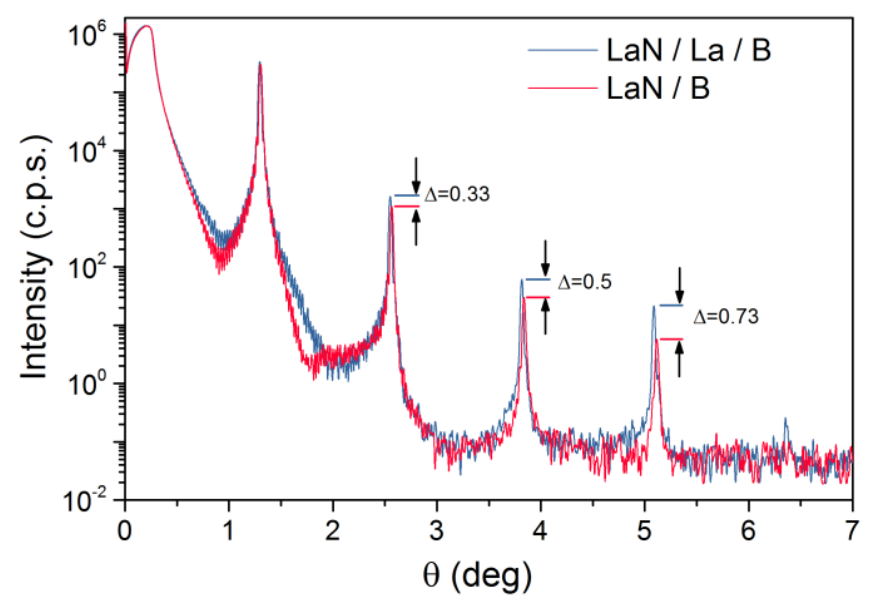

Figure 4 GIXRR of a conventional LaN/B multilayer (red curve) and modified LaN/La/B multilayer with a 0.3 La interlayer above B layer (blue curve).
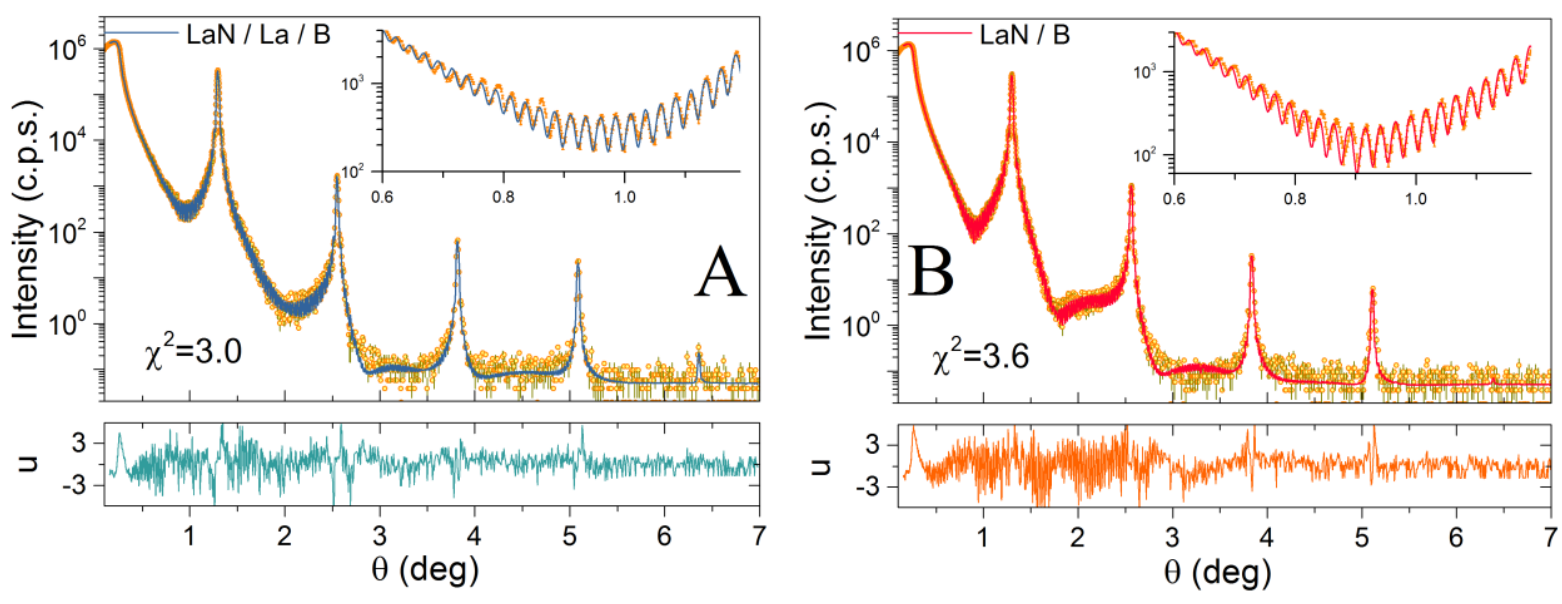

Figure 5 Fit of the experimental GIXRR curves for (a) LaN/B and (b) LaN/La/B multilayers. Calculated curves are shown in solid lines, experimental curves in yellow points, bottom subplots show residuals between calculated and experimental curves. 
The whole manifold of the $\delta$-profiles for all the solutions is presented in Fig. 6. According to equation (1) the solutions with 11 sublayers should have an optimal number of sublayers, therefore they were used to calculate the error corridors of determination of $\delta$, using the approach based oncorrelation analysis of uncertainties from (Yakunin et al., 2014). These corridors are also present in Fig. 6, and the differences between the individual $\delta$-profiles in each manifold fit in the error corridor.

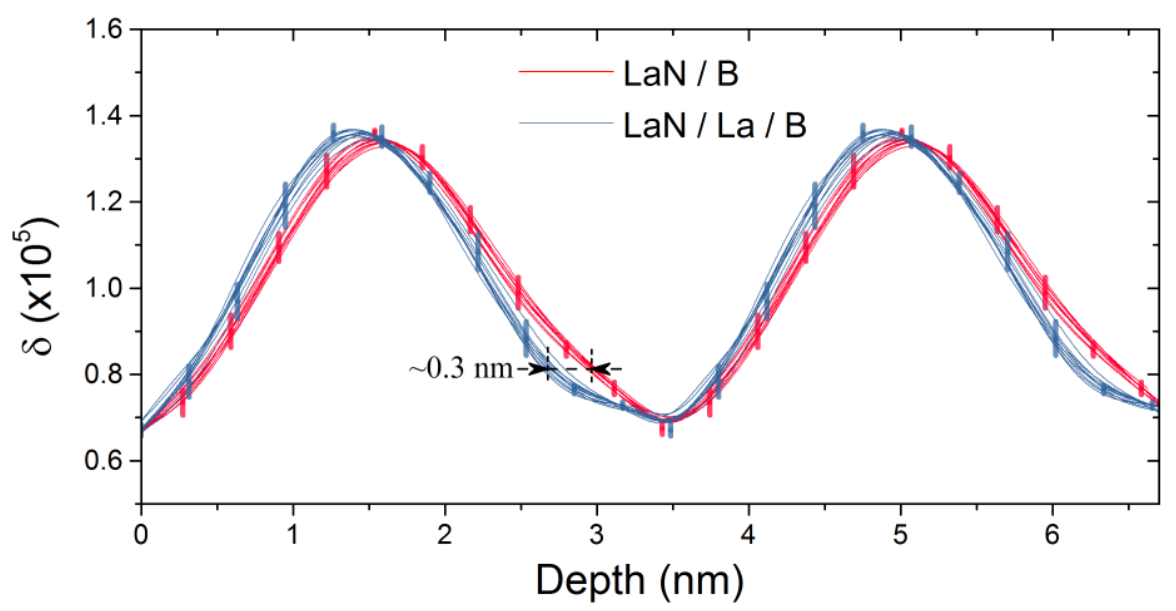

Figure 6 The multiple $\delta$-profiles obtained from the fitting procedure for $\mathrm{LaN} / \mathrm{B}$ and $\mathrm{LaN} / \mathrm{La} / \mathrm{B}$ structures with $0.3 \mathrm{~nm}$ La interlayers. Two consecutive periodic parts are shown. Error corridors for profiles with 11 sublayers are added.

The major distinction of the solutions shown in Fig. 6 from the ones shown in Fig. 2 is that for each experimental set only one group of reconstructed profiles was found. We believe this is connected to a much smaller asymmetry of interfaces in the given profiles, compared to the profiles from Fig. 2A.

Next we discuss the features of the reconstructed profiles for LaN/B and LaN/La/B experimental multilayers (Fig 6). The major noticeable difference in these profiles is found at the beginning of the La-on-B interface. This is in a good agreement with the position of the expected reduced B-N interaction at the LaN-on-B interface due to the $0.3 \mathrm{~nm}$ La interlayer introduced. Apparently, the reduced formation of the boron nitride at that interface makes the top part of B layer more pure hence lowering the $\delta$ region at the very beginning of the La-on-B interface. In this area the difference between the reconstructed profiles for two samples is around $0.3 \mathrm{~nm}$, which correlates well with approximate $0.3 \mathrm{~nm}$ increase of pure B thickness found in a separate investigation (Kuznetsov, 2016). The introduction of the $0.3 \mathrm{~nm}$ La interlayer also resulted in a small shift of the LaN with respect to the B layer, so that the maxima of the profiles do not coincide, while the minima are aligned. This may be explained by different compaction of formed compound(s) at the La-on-B interface. These two features are apparently the cause of the differences observed in the GIXRR curves. Although the differences seem to be small at $0.154 \mathrm{~nm}$ wavelength, the BEUV reflectance increases from 58 to $64 \%$, because the BN is optically unfavourable on top of B layer in the BEUV region. 
As shown above the considered technique is sufficiently sensitive for small differences in the structure. However, concerning the analysis of real samples it is important to have only small thickness deviations between the individual layer periods. If present, they will result in a broadening of the high order Bragg peaks. These aperiodic effects cannot be included in the model while keeping a periodic condition, and the optimization algorithm will try to compensate for these features by changing the structure. In both given structures these effects are not significant, although the $4^{\text {th }}$ Bragg peak of reference sample is still slightly broader than the calculated one. So attention should be paid not only to the quality of the GIXRR measurement, but also to the quality of the multilayered sample itself.

Finally it is important to notice that the obtained $\delta$-profiles have almost no flat part around the LaN or $\mathrm{B}$ regions. It would be very challenging and time-consuming to find similar quality solutions with a conventional model approach, which would require multiple manual restarts to adjust the model. The free-form approach can do this job in one run, provided that the adequate parameters (the optimal amount of sublayers and the parameters of instrumental function: angular resolution, background) for a given quality of measurement are used. This makes it much easier tool for routine analysis of different multilayers and comparison of their structures.

\section{Conclusions}

Conventional model approaches for the structural reconstruction from GIXRR measurements of layered structures are generally lacking flexibility when dealing with very thin structures, because assumptions have to be made about the interface structure. It is almost impossible for these techniques to precisely describe GIXRR of the short period multilayers, for example, $\mathrm{La} / \mathrm{B}$-based BEUV mirrors. In this work we presented the first attempt of a free-form approach for the reconstruction of the optical constant profile of periodic multilayer structures based on a rigorous dynamic description of the X-ray reflectivity.

At first, the approach was validated on simulated data to show that reconstruction of a given profile is possible within the resolution of the technique used. This demonstrated very good $\delta$-profile reconstruction and GIXRR curve matching for the simulated data. At the same time the problem of multiple solutions inherent to inverse problem of X-ray reflectometry was analysed.

The $\delta$-profiles of two magnetron deposited multilayers, $\mathrm{LaN} / \mathrm{B}$ and $\mathrm{LaN} / \mathrm{La} / \mathrm{B}$, were reconstructed and showed good matching of the measured GIXRR curves. The differences between two structures showed that an introduction of a $0.3 \mathrm{~nm}$ La can indeed improve the optical contrast at the LaN-on-B interface, as was initially suggested. The obtained $\delta$-profiles did not contain a flat part around LaN or $\mathrm{B}$ regions, indicating a gradient, non-pure composition at these minima/maxima. This type of 
structure would be very difficult to reconstruct using any conventional model approach. At the same time it was demonstrated that the free-form approach applied in this work can do this job in one run. As such this makes it a much easier tool for routine analysis of multilayers and comparison of their structures.

Acknowledgements We acknowledge the support of the Industrial Focus Group XUV Optics at the MESA+ Institute at the University of Twente, notably the industrial partners ASML, Carl Zeiss SMT AG, PANalytical, SolMates, TNO, and Demcon, as well as the Province of Overijssel and the Foundation FOM.

\section{References}

Banine, V., Yakunin, A., \& Glushkov, D. (2010). 2010 Int. Work. Extrem. Ultrav. Sources. 14.

Chou, C.-H., Regan, M. J., Pershan, P. S., \& Zhou, X.-L. (1997). Phys. Rev. E. 55, 7212-7216.

Daillant, J. \& Gibaud, A. (2009). X-ray and Neutron Reflectivity Berlin, Heidelberg: Springer Berlin Heidelberg.

Jergel, M., Holý, V., Majková, E., Luby, Š., Senderák, R., Stock, H. J., Menke, D., Kleineberg, U., \& Heinzmann, U. (1998). Phys. B Condens. Matter. 253, 28-39.

Kozhevnikov, I. V. (2003). Nucl. Instruments Methods Phys. Res. Sect. A Accel. Spectrometers,

Detect. Assoc. Equip. 508, 519-541.

Kuznetsov, D. S. (2016). Personal communication.

Kuznetsov, D. S., Yakshin, A. E., Sturm, J. M., van de Kruijs, R. W. E., Louis, E., \& Bijkerk, F. (2015). Opt. Lett. 40, 3778.

Makhotkin, I. A. (2013). Structural and reflective characteristics of multilayers for 6.x nm wavelength. University of Twente.

Makhotkin, I. A., Zoethout, E., Louis, E., Yakunin, A. M., Müllender, S., \& Bijkerk, F. (2012). Opt. Express. 20, 11778-11786.

Pedersen, J. S. (1992). J. Appl. Crystallogr. 25, 129-145.

Pershan, P. S. (1994). Phys. Rev. E. 50, 2369-2372.

Platonov, Y. Y., Gomez, L., \& Broadway, D. (2002). Proc. SPIE 4782, X-Ray Mirrors, Crystals, and Multilayers, A.K. Freund, A.T. Macrander, T. Ishikawa, \& J.L. Wood, edited by, pp. 152-159.

Sanyal, M. K., Sinha, S. K., Gibaud, A., Huang, K. G., Carvalho, B. L., Rafailovich, M., Sokolov, J., Zhao, X., \& Zhao, W. (1993). Europhys. Lett. 21, 691-696.

Tolan, M. (1999). X-ray scattering from soft matter thin films: material science and basic research Springer-Verlag Berlin Heidelberg.

Tsarfati, T., van de Kruijs, R. W. E., Zoethout, E., Louis, E., \& Bijkerk, F. (2009). Thin Solid Films. $518,1365-1368$. 
Windt, D. L. (1998). Comput. Phys. 12, 360-370.

Yakshin, A., Louis, E., Görts, P., Maas, E. L. G., \& Bijkerk, F. (2000). Phys. B. 283, 143-148.

Yakunin, S. N., Makhotkin, I. A., Nikolaev, K. V., van de Kruijs, R. W. E., Chuev, M. A., \& Bijkerk, F. (2014). Opt. Express. 22, 20076.

Zhou, X.-L. \& Chen, S.-H. (1993). Phys. Rev. E. 47, 3174-3190.

Zimmermann, K.-M., Tolan, M., Weber, R., Stettner, J., Doerr, a., \& Press, W. (2000). Phys. Rev. B. 62, 10377-10382. 